\title{
Linx
}

Revue des linguistes de l'université Paris X Nanterre

48 | 2003

Approches syntaxiques contemporaines

\section{Bresnan, Joan (2001) Lexical-Functional Syntax}

Oxford, Blackwell Publishing, 446 p., $19 £$

\section{Danielle Leeman}

\section{CpenEdition}

Journals

Édition électronique

URL : http://journals.openedition.org/linx/239

DOI : $10.4000 / \operatorname{lin} \times .239$

ISSN : 2118-9692

Éditeur

Presses universitaires de Paris Nanterre

\section{Édition imprimée}

Date de publication : 1 juin 2003

Pagination : 165-167

ISBN : 0246-8743

ISSN : 0246-8743

Référence électronique

Danielle Leeman, «Bresnan, Joan (2001) Lexical-Functional Syntax», Linx [En ligne], 48 | 2003, mis en ligne le 01 octobre 2003, consulté le 22 septembre 2020. URL : http://journals.openedition.org/linx/ 239 ; DOI : https://doi.org/10.4000/linx.239 


\section{Bresnan, Joan (2001) Lexical-Functional Syntax, Oxford, Blackwell Publishing, 446 p., $19 £$}

L'ouvrage se présente comme une introduction à la syntaxe lexicalefonctionnelle, qui a émergé à la fin des années soixante-dix à l'initiative de Joan Bresnan elle-même, en opposition, comme beaucoup de formalismes contemporains, au modèle élaboré par Noam Chomsky et ses disciples en tant qu'il se veut une «grammaire universelle » : une langue qui ne fonctionnerait pas de la façon que cette grammaire le prédit pour toute langue constituerait évidemment un contre-exemple qui falsifierait le modèle et conduirait à en définir un autre ${ }^{4}$. C'est le cas en l'occurrence: la contestation concerne la représentation du fonctionnement de la phrase en termes d'une structure syntaxique hiérarchisée (schématisée par l'arbre), qui suppose que l'énoncé s'organise selon les relations qui s'établissent entre des groupes (ou constituants) correspondant à des unités conceptuelles. La phrase en warlpiri (langue aborigène, Australie) ne s'organise en effet pas selon des groupes syntaxiques, si bien que différents ordres linéaires sont possibles, contrairement à ce qui se passe en anglais : l'équivalent de $*$ The two small are chasing that children dog est ainsi aussi possible dans cette langue que celui de The two small cbildren are chasing the dog, du fait que l'essentiel des relations grammaticales y est marqué morphologiquement. Cela ne veut pas dire que le warlpiri ne puisse pas exprimer les mêmes informations que l'anglais (et réciproquement) - ainsi qu'en témoigne l'exemple précédent - mais que le sens se véhicule, selon les langues, plutôt par les mots ou plutôt par les syntagmes, l'importance de la morphologie étant inversement proportionnelle à celle de la syntaxe (et réciproquement). Cette différence d'organisation dans l'expression n'empêche pas en effet que se retrouvent, sous les deux modalités formelles, les mêmes unités conceptuelles ("objets», "événements», "participants d'un événement», «états psychologiques ", etc.) et les mêmes contraintes grammaticales : en anglais comme en warlpiri - mais sous des formes différentes - un pronom réfléchi ne peut être qu'un complément coréférentiel du sujet (Pauli a honte de lui $i_{i}$ et non un sujet coréférentiel du complément (* Lui a honte de Pauli). L'universalité est donc à rechercher non dans la structure phrastique mais dans les relations grammaticales sous-jacentes aussi bien à la forme syntaxique (pour l'anglais) qu'à la forme morphologique (pour le warlpiri), ce que la Grammaire Lexicale-Fonctionnelle trouve dans la structure argumentale des prédicats : en écho à l'exemple donné initialement, le point commun à l'anglais et au warlpiri est donc chase < agent, patient >.

Dans l'appellation "Grammaire Lexicale-Fonctionnelle », l'adjectif lexicale est par conséquent là pour marquer l'importance des mots (en tant qu'unités lexicales)

périphérique par rapport à la doxa, mais plus directement accessible et muni d'exercices avec leurs corrigés : Delaveau, A. (2001) Syntaxe. La phrase et les subordinations, Paris, Colin, coll. "Campus», $192 \mathrm{p}$.

${ }^{4}$ C'est aussi ce qui motive explicitement l'élaboration des grammaires de construction - cf. dans le présent numéro de Linx la contribution de Y. Mathieu. De même la « Role \& Reference Grammar » s'inscrit-elle en faux contre la thèse de l'autonomie de la syntaxe - cf. l'article de J. François icimême. 
dans l'expression des relations grammaticales, et l'adjectif fonctionnelle marque que ces relations grammaticales ne sont pas issues d'une organisation syntaxique ou morphologique mais d'un système de correspondances entre les prédicats et leurs arguments d'une part, et les événements qu'ils dénotent d'autre part. Concrètement, la grammaire doit relier la structure argumentale (de type : verbe $<\mathrm{x}, \mathrm{y}>$ ), la structure fonctionnelle (de type : SUJET ... OBJET ... PREDICAT ...) et la structure catégorielle (le prédicat est un $\mathrm{V}$, le sujet est un $\mathrm{SN}$, etc.). La structure catégorielle concerne l'expression (la forme elle-même) et varie selon les langues (ainsi, on ne peut dans certaines langues définir de constituant $\mathrm{GV}$, contrairement à ce qui se produit en anglais) : dans les exemplifications ici retenues, elle emprunte à la théorie $\mathrm{X}^{\prime}$ de la grammaire générative et aux représentations arborescentes. La structure fonctionnelle en revanche est conçue comme invariante et commune à toutes les langues (contrairement à la notion syntaxique de « GV », celle de « PRED » est universelle - à condition de ne pas l'assimiler à telle ou telle actualisation morphologique ou syntaxique). La structure argumentale relève à la fois du sens et de la forme : elle représente en effet les participants définitoires du procès (cf. ci-dessus : chase < agent, patient $>$ ), autrement dit décrit la structure de l'événement lui-même, mais en même temps elle représente les éléments syntaxiquement dépendants d'une tête (l'élément précisément susceptible d'avoir des arguments, en l'occurrence : le verbe).

Après avoir, dans une première partie, motivé par divers exemples le bienfondé d'une grammaire différente de celle que défendent les générativistes, l'auteur en présente dans une deuxième partie l'architecture formelle, qui repose sur un modèle mathématique, la justification en étant que, si les formalismes apparaissent beaucoup trop simples et grossiers («crude ») pour rendre compte de la réalité empirique, ils ont en revanche l'avantage de permettre des analyses univoques et des généralisations vérifiables ${ }^{5}$. La troisième partie s'attache à montrer le fonctionnement du modèle : comment sont mises en correspondance les trois structures précédemment évoquées ; chacune des fonctions (de la structure fonctionnelle) relie les composantes de la structure argumentale à ceux de la structure catégorielle, de sorte qu'un ensemble de formes est défini comme relevant de telle fonction, selon leur type de connexion avec tel argument: il peut par exemple pour le SUJET s'agir, selon les langues, de l'argument qui joue le rôle d'agent, ou de celui qui au contraire joue le rôle de patient (comme dans les langues ergatives). La quatrième partie est consacrée aux relations grammaticales plus «abstraites » (i.e. non formellement explicitées), comme celles qui concernent le comportement et l'interprétation des pronoms: comment rendre compte du fait que l'on ait (l'indice $i$ marquant la coréférence) Léa a bonte d'elle-même mais non * Léai a bonte d'elle $\mathrm{ni}^{*}$ Elle (-même) i a bonte de Léa ${ }_{i} \mathrm{ni} *$ Le père de Léa a honte d'elle-mêmei, etc. L'ouvrage se clôt par un ensemble de problèmes et leurs solutions mais non justifiées : celui qui n'arrive pas au «bon » résultat devra lui-même trouver pourquoi! La bibliographie, outre les références mentionnées dans le corps du texte, renseigne sur l'ensemble des travaux publiés dans le champ. Un index des notions occupe les cinq dernières pages.

Je mentirais en écrivant que l'ouvrage est accessible aux néophytes: contrairement à celui d'A. Carnie (cf. compte rendu précédent), il suppose une culture

${ }^{5}$ Cf. la contribution de M. Cori dans le présent numéro de Linx. 
aussi bien linguistique (en particulier dans le domaine de la grammaire générative) que mathématique, et intéressera donc plutôt des étudiants ou des chercheurs déjà familiarisés non seulement avec les problèmes récurrents de la grammaire (anaphores, compléments et ajouts, etc.) mais encore avec d'autres modèles syntaxiques. Cela posé, on dispose là d'une présentation claire et élégante, intégrant de manière naturelle et progressive à l'explicitation des principes théoriques les résultats essentiels des différents chercheurs travaillant dans ce cadre, et donc extrêmement précieuse pour la connaissance des sciences du langage aujourd'hui.

\section{Muller, Claude (2002) Les bases de la syntaxe. Syntaxe contrastive français- langues voisines, Pessac, Presses Universitaires de Bordeaux, 454 p. $30 €$}

L'ouvrage se présente comme une initiation à la linguistique centrée sur la syntaxe, celle-ci définie comme «le domaine des combinaisons, des choix, des associations d'unités significatives » (p. 12), dont l'objectif est à la fois de présenter « un corps de connaissances et d'hypothèses» (p. 11) sans exclusive d'école, sur des thématiques diverses (les constituants de la phrase) mais articulées entre elles, et incluant une comparaison avec différentes langues (anglais, espagnol, italien, allemand, basque, breton, portugais, gascon, voire finnois). L'auteur adopte une "perspective dérivationnelle » (p. 18), cherchant à retrouver sous les énoncés (qui ne sont que l'effet tangible d'une mise en œuvre préalable) les différentes étapes de leur production. L'hypothèse fondamentale présidant à la forme générale de la grammaire ici proposée est que l'objet de la syntaxe ne peut être borné aux hiérarchies structurales, du fait que «tout énoncé résulte de plusieurs structurations différentes ayant chacune leur logique » (p. 20), qui concernent pour l'une les prédicats et leurs arguments, une autre l'organisation en syntagmes, une troisième la répartition de l'information (thème, rhème, focus), et le tout un soubassement sémantique « dès le départ de la construction de l'énoncé » (p. 22) ${ }^{6}$. La syntaxe au sens banal (i.e. le domaine de la structure) est ce qui «forme l'essentiel de la grammaire d'une langue donnée, avec ses particularités » (p. 73), par opposition au niveau prédicatif, qui est universel (ibid.) ${ }^{7}$; elle est en effet largement tributaire du sens puisque c'est la visée énonciative (l'intention illocutoire et l'information à transmettre) qui détermine le choix des unités lexicales propres à véhiculer ce que l'on veut exprimer, et que ce sont les unités lexicales qui déterminent en grande partie la structure de la phrase : les mots ne sont pas à concevoir comme des formes morphologiques simplement munies d'une identité sémantique, ils sont

\footnotetext{
${ }^{6}$ On retrouve ici l'hypothèse guillaumienne, précédant celle d'A. Culioli, ou, plus récemment et hors de France, des fonctionnalistes tels que S. Dik ou T. Givón (ces derniers mentionnés par Claude Muller) - cf. dans le présent numéro de Linx la contribution de Jacques François.

${ }^{7}$ C'est aussi ce qui motive l'élaboration par J. Bresnan d'une grammaire lexico-fonctionnelle (cf. le compte-rendu précédent), par opposition à la syntaxe universelle prônée par le courant générativiste.
} 